Correspondence to: Dr Kwai Fu Ko, Block 5, Flat D, 19/F, City Garden, 233 Electric Road, North Point, Hong Kong.

1 John TR, Crowleg WJ, Miller JQ, et al. The syndrome of myasthenia and polymyositis with comments on therapy. Ann NY Acad Sci 1971;183:64-71

2 Kikuchi H, Hayashi N, Nakajima T, et al. A case report of autoimmune hepatitis with myasthenia gravis. Nippon Shokakibyo Gakkai Zasshi 1988;85:2261-4

3 Kirk AP, Jain S, Pocock S, et al. Late results of Royal Free Hospital controlled trial of prednisolone therapy in hepatitis B surface antigen-negative chronic active hepatitis. Gut 1980;21:78-83.

4 Ehrenstein MR, Snaith ML, Isenberg DA. Idiopathic myositis: a rheumatological view. Ann Rheum Dis 1992;51:41-4.

5 Garlepp M J. Immunogenetics of inflammatory myopathies. Bailliere's Clinical Neurology 1993;2:579-97.

\section{Transient monocular blindness}

The term amaurosis fugax, which means "fleeting blindness", has come to be associated with transient monocular blindness due to emboli reaching the retinal circulation from the carotid vessels or from the heart. As amaurosis fugax may precede a stroke it is usually viewed in a neurological context. Transient monocular blindness, however, is not solely caused by emboli. It has many causes, including migraine, intracranial hypertension, and malignant hypertension and it is, therefore, important to make an accurate diagnosis. We describe a common ophthalmic condition, intermittent angle closure glaucoma, giving rise to transient monocular blindness, so emphasising the need to be aware of such diagnoses.

A 66 year old woman presented with a 12 month history of repeated episodes of transient loss of vision in her right eye. The episodes were precipitated by reading, writing, and watching television for a variable time. They came on suddenly and she described a film descending over her right eye leading to complete loss of vision. The episodes lasted from three minutes to several hours. On occasions there was an associated ache over the right side of the forehead. She had no positive visual symptomatology during an attack and there was no family history of migraine. She smoked six cigarettes a day.

She had a history of palpitations and blackouts for which she attended a cardiologist. An ECG and an exercise stress test were normal; however, a 24 hour Holter monitor had disclosed frequent ventricular extrasytoles and an episode of atrial fibrillation. She was treated with a $\beta$ blocker and subsequently amiodarone, but this was discontinued by the patient. Neurological examination was normal, as were carotid duplex scans, brain CT, and an echocardiogram. A provisional diagnosis of amaurosis fugax was made and she was started on $75 \mathrm{mg}$ aspirin a day. In view of the specific precipitating factors of her visual loss, she was referred for a neuro-ophthalmic opinion.

Initial neuro-ophthalmic assessment was normal. To assist in reaching a diagnosis it was decided to try to precipitate an attack. After reading intermittently over a period of four hours she reported loss of vision in her right eye. Slit lamp examination showed pronounced corneal oedema on the right, a poorly reacting semidilated pupil and a shallow anterior chamber. Her intraocular pressures were $50 \mathrm{~mm} \mathrm{Hg}$ on the right and
$18 \mathrm{~mm} \mathrm{Hg}$ on the left. Pulsation of the central retinal artery was noted on the right.

A diagnosis of intermittent angle closure glaucoma was made. After initial medical treatment to constrict the pupil and lower the intraocular pressure, nd-YAG laser iridotomies were performed. Subsequent gonioscopy confirmed a narrow drainage angle and refraction disclosed a moderate degree of hypermetropia which may be associated with a shallow anterior chamber and narrow drainage angle. At a review appointment three months later, the patient reported that since the iridotomies she had had no further episodes of visual disturbance.

The first report that transient monocular blindness could precede contralateral hemiplegia was by Miller Fisher in $1952 .{ }^{1} \mathrm{He}$ stated that "Blindness is usually complete in the affected eye, although at times the defect is limited to one sector. The frequency of attacks varies from several a day to a few each year. Symptoms last for years or may disappear completely after a few months...The blindness most commonly comes on as though a blind were being lowered or raised, and vision returns from the opposite direction... The attacks last from a minute or so up to seven minutes or more." This led to the awareness that transient monocular blindness may warn of an impending stroke and the need to institute preventative measures.

Our patient had many of the features reported by Miller Fisher. She also had several cardiovascular risk factors, being a smoker and having a history of cardiac arrhythmias. She had features which suggested an alternative diagnosis, however. In particular the specific precipitating factors were atypical. The duration of attacks, which on occasions lasted several hours, was unusual, although attacks of amaurosis fugax of up to 24 hours have been reported. ${ }^{2}$

A diagnosis of angle closure glaucoma was not originally suspected, as on direct questioning the patient neither reported seeing haloes, nor having any visual loss associated with poor lighting conditions. Angle closure glaucoma is usually associated with severe pain and injection of the globe, whereas our patient had complained only of an aching sensation and had not noted any redness of the eye.

Angle closure glaucoma has previously been reported as a cause of transient monocular visual loss. ${ }^{3}$ In the three cases reported the initial presenting diagnoses were either of amaurosis fugax or migrainous phenomena. Two of the patients described seeing haloes, however, and none of them had specific precipitating factors such as reading. Close ocular work such as reading and sewing can precipitate angle closure glaucoma. It is, however, uncommon for reading to be the sole precipitating factor.

Angle closure glaucoma can cause visual loss by various different mechanisms. The raised intraocular pressure leads to corneal clouding due to oedema and may reduce the perfusion pressure of the eye, thereby impairing blood flow to the choroid, retina, and optic disc. 4

In conclusion, although emboli are responsible for most cases of transient monocular blindness other causes should always be considered (see review by Gautier ${ }^{5}$ ), especially in the presence of atypical features such as specific precipitating factors or an unusually long duration of symptoms.
EOIN O'SULLIVAN SANDIP SHAUNAK TIMOTHY MATTHEWS CHRISTOPHER KENNARD Academic Unit of Neuroscience, Medical School, London, UK PETER SIMCOCK

Charing Cross and Westminster Medical Scho
London, UK
PETER SIMCOCK

Hospital, London, UK

Correspondence to: Professor C Kennard, Academic Unit of Neuroscience, Charing Cross and Westminster Medical School, Fulham Palace Rd, London W6 8RF, UK.

1 Fisher CM. Transient monocular blindness associated with hemiplegia. Arch Ophthalmol 1952;47:167-203.

2 Parkin PJ, Kendall BE, Marshall J, McDonald WI. Amaurosis fugax: some aspects of management. 7 Neurol Neurosurg Psychiatry 1982. 45:1-6.

3 Ravits J, Seybold ME. Transient monocular loss from narrow angle glaucoma. Arch loss from narrow an

4 Best M, Blumenthal M, Futterman HA, Galin MA. Critical closure of intraocular blood MA. Critical closure of intraocular blood
vessels. Arch Ophthalmol 1969;82:385-92.

5 Gautier J-C. Clinical presentation and differential diagnosis of amaurosis fugax. In: Bernstein EF, ed. Amaurosis fugax. New York: Springer-Verlag, 1988:24-42.

Acquired bilateral opercular lesions or Foix-Chavany-Marie syndrome and eating epilepsy

The Foix-Chavany-Marie syndrome or bilateral anterior opercular syndrome (AOS) consists of lower facial and glossopharyngeal diplegia secondary to dysfunction of the rolandic opercula. It is usually seen in adults. It can result from tumour or infection but is mainly due to successive strokes involving both opercular regions. ${ }^{1}$ Children developing AOS acutely as a consequence of an acute CNS illness have been described. ${ }^{2}$

A 20 year old man had normal development until, at the age of 4 years, he developed acute, presumably viral, meningoencephalitis. This led to a prolonged stay in hospital in an intensive care unit. He was left with severe dysarthria, dysphasia, and bilateral labioglossopharyngeal paresis. At the age of 11 he developed partial motor seizures (involving the left facial muscles) with occasional secondary generalisation. Attacks occurred only during meals and there was no relation with any specific foods, definite times, or other specific triggering factors. He had received different antiepileptic regimens with poor results until combined treatment with carbamazepine $(600 \mathrm{mg} /$ day $)$, valproate $(1500 \mathrm{mg} /$ day $)$, and clobazam $(15 \mathrm{mg} /$ day) was initiated. He now has only one or two seizures a year, always triggered by eating.

On neurological examination his mouth was always open and he drooled continuously. He had bilateral lip, tongue, and pharyngeal weakness with dissociation of automatic and voluntary movements of the lower face (voluntary movements impaired and automatic movements preserved). Eye closure and extraocular movements were normal. Affect was normal. He had a brisk jaw jerk. Language was limited to guttural vowel sounds, but his comprehension was normal. He had a minimal left upper limb paresis and generalised hyperreflexia, more pronounced on the left. Computed tomography and MRI showed atrophic lesions involving both rolandic opercula (figure). Recordings from EEG showed normal back- 


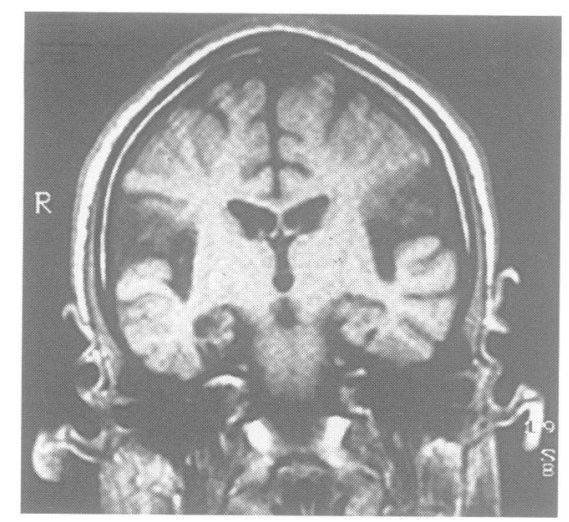

Patient with acquired bilateral opercular syndrome and eating epilepsy: coronal T1 weighted MRI showing focal atrophy in both rolandic opercula.

ground activity with focal interictal slow spikes and waves over the right centrotemporal region, not increased during sleep. No ictal recording was obtained.

Voluntary control of facial muscles is dependent on the normal function of the motor cortex and of the corresponding pyramidal tract. Subcortical structures, particularly the basal ganglia, thalamus, and subthalamic nuclei, are involved in automatic or emotional movement. A bilateral lesion involving the anterior opercular region is manifested by facial diplegia with dissociation of voluntary and involuntary movements. ${ }^{1}$ Pyramidal signs, cheiro-oral paresthaesiae, and severe speech disturbance or even mutism with unimpaired language comprehension may be due to or associated with bilateral opercular lesions depending on their symmetry and extent. ${ }^{1}$ Symmetric lesions of the posterior limbs of both internal capsules may produce a picture similar to AOS because the projections from the anterior opercular cortex are represented there. The differential diagnosis of AOS, therefore, is based not only on bulbar dysfunction but also on other clinical features of pseudobulbar palsy, such as those due to striate or pontine lesions. ${ }^{3}$ The absence of automaticvoluntary dissociation and affective lability point to the presence of AOS. ${ }^{1}$ Bilateral stroke is the main aetiology of AOS and the two events may be separated in time. A unilateral lesion may lead to AOS in a patient who already has contralateral opercular dysfunction on the other side.

The occurrence of AOS in childhood is exceptional. In most cases the syndrome is of developmental origin with polymicrogyria most likely due to prenatal vascular lesions. Infections of the CNS are also an important cause of the syndrome in children. ${ }^{2}$ There are well documented cases with transient bilateral rolandic dysfunction and features resembling AOS due to an epileptic disturbance. ${ }^{4}$

In this patient, reflex seizures were triggered by a specific stimulus, the act of eating. Eating epilepsies have been subclassifled into two distinct groups with either temporal or opercular onset, according to a recent anatomoclinical review. ${ }^{5}$ In the opercular subgroup electroclinical studies pointed to the presence of an epileptogenic focus in the postcentral gyrus, frequently with evidence of a cortical structural lesion at that level. The physical characteristics and the type of food seem to be important factors in activating this form of eating epilepsy. Attacks may recur even when temporal structures and those anterior or posterior to the sensory motor strip have been resected. In addition to reflex seizures, spontaneous attacks may occur. Thalamic sensory afferents to the damaged cortex seem to play a predominant part in the genesis of seizures in these patients. By contrast, eating epilepsy of temporal origin is dependent more on the context of a meal and there are often additional clinical features suggesting temporal localisation. Such patients have no features of a postcentral lesion and a temporal resection usually leads to cessation.

Our patient clearly showed an AOS. $\mathrm{He}$ had no spontaneous seizures and we were not able to confirm that the characteristics of the food played a part in triggering the attacks. He is exceptional because of the association of two infrequent but related neurological syndromes. We stress the value of careful imaging and of inquiring about the circumstances of occurrence of attacks in diagnosing this syndrome and the associated reflex epilepsy.

V MATEOS
J SALAS-PUIG
D M CAMPOS
Neurological Department,
Hospital General de Asturias,
Oviedo,
Spain
V CARRERO
Internal Medicine Department,
Hospital de Mérida,
Spain
F ANDERMANN
Montreal Neurological Hospital and Institute,
Montreal,
Canada

Correspondence to: Dr V Marcos, Servicio de Neurologia, Hospital General de Asturias, 33006Oviedo, Spain.

1 Mao CC, Coull BM, Golper LAC, Rau MT. Anterior operculum syndrome. Neurology 1989,39:1169-72.

2 Grattan-Smith PJ, Hopkins IJ, Shield LK, Boldt DW. Acute pseudobulbar palsy due to Boldt DW. Acute pseudobulbar palsy due to
bilateral focal cortical damage: The opercubilateral focal cortical damage: The opercular syndrome of Fox-Ch

3 Besson G, Bogousslavsky J, Regli F, Maeder P. Acute pseudobulbar or suprabulbar palsy. Arch Neurol 1991;48:501-507.

4 Colamaria V, Sgro V, Caraballo R, et al. Status epilepticus in benign rolandic epilepsy manifesting as anterior operculum syndrome. Epilepsia 1991;32:329-334.

5 Rémillard GM, Andermann F, Zifkin BG, Olivier A, Rasmussen T. Eating epilepsy. A study of ten surgically treated patients suggests the presence of two separate syndromes. In: Beaumanoir A, Gastaut $\mathrm{H}$, Naquet R. eds. Reflex seizures and reflex epilepsies. Gene

HTLV 1 associated myelopathy and adult $T$ cell leukaemia-lymphoma in the same patient: report of a case

Tropical spastic paraplegia (TSP) is a chronic progressive myelopathy occurring in endemic areas around the world with particularly high prevalence in Japan, the Caribbean, parts of Africa, and South America. In 1985, Gessain and colleagues working on the island of Martinique identified the presence of anti-HTLV 1 antibodies in patients with TSP. ${ }^{1}$ This finding was later confirmed in other tropical regions where this condition is endemic. In Japan, Osame et al called it HTLV 1 associated myelopathy $(\mathrm{HAM}) .^{2}$ The HTLV 1 retrovirus had previously been isolated and identified in 1980 by Poiesz et al as the aetiological agent of adult $\mathrm{T}$ cell leukaemia-lymphoma (ATLL). ${ }^{3}$ Epidemiological studies have since shown the high prevalence of both ATLL and HAM/TSP in areas with a high HTLV 1 carrier rate. The virus has been isolated from patients with both the conditions and has been shown to be the same strain of the retrovirus. It is not known why some carriers develop chronic progressive myelopathy whereas others develop the haematological malignancy. The coincidence of both conditions occurring together as in our patient is extremely rare.

A 61 year old black Afro-Caribbean woman who had migrated to the United Kingdom from the Dominican Republic was seen with a four year history of progressive difficulty in walking and backache. She had also noticed a progressive deterioration in her vision and difficulty in micturation. Two and a half years later she was found to have a positive syphilis serology. A CSF examination was normal.

Neurological examination on presentation disclosed diminution of visual acuity in both eyes (N9 near vision) and the fundus examination showed bilateral optic atrophy. The other cranial nerves were normal. She had evidence of a spastic paraparesis, with a pyramidal distribution of weakness in the legs, exaggerated reflexes, and extensor plantar responses.

The tone and power in her arms were normal, although the reflexes were brisk. Sensory examination was normal to all modalities and there were no cerebellar signs. She was able to walk only with the help of a stick and she had a spastic gait.

Haemoglobin concentration was $10 \mathrm{~g} / \mathrm{dl}$ and the white cell count was $10.2 \times 10^{9} / 1$. Magnetic resonance imaging of the cervical and dorsal spine was normal but MRI of the brain showed hyperintense signals in the periventricular white matter on T2 weighted images. Visual evoked responses were delayed bilaterally and the somatosensory evoked responses showed delay of N19 on stimulation of the median nerves at the wrists bilaterally. Antibodies to HTLV 1 were detected in the serum and a diagnosis of HTLV 1 associated myelopathy (HAM) was made.

Four months after the diagnosis of HAM, the patient had a rapid deterioration of power in her legs. She was febrile and delirious. She became progressively obtunded and her level of consciousness rapidly worsened. There was considerable tenderness in the long bones. The liver and spleen could not be palpated. Haemoglobin was $10 \mathrm{~g} / \mathrm{dl}$, and the white cell count was $66 \times 10^{9} / 1$. The peripheral smear showed a pronounced lymphocytosis and presence of abnormal lymphocytes with multilobulated nucleii with clover and cleaved forms. About $20 \%$ of the lymphocytes showed abnormal multilobulated nucleii. Leukaemia typing confirmed that the abnormal cells were all $\mathrm{T}$ lymphocytes (CD-2 count 98\%, CD-3 count $97 \%$, CD-4 count $76 \%$, and CD-5 count $0 \%$ ) and a diagnosis of adult $T$ cell leukaemia was made. Examination of CSF, skeletal survey, and serum calcium concentrations were normal. The patient received supportive treatment but she soon deteriorated and died. 